\title{
Temperature Dependence of Dielectric Constant with Different Poling Fields in Lead Magnesium Niobate-Lead Titanate Single crystal
}

\author{
Noriko Yamamoto, Yohachi Yamashita*, Kazuhiro Itsumi and Yasuharu Hosono \\ Corporate Research and Development Center, Toshiba Corpotation, 1 Komukai-Toshiba-cho, Saiwai-ku, Kawasaki 212-8582, \\ Japan, \\ Fax: 81-44-520-1255, e-mail: norikok.yamamoto@toshiba.co.jp, kazuhiro.itsumi@toshiba.co.jp, yasuharu.hosono@toshiba.co.jp \\ *Toshiba Research Consulting Corporation, 1 Komukai-Toshiba-cho, Saiwai-ku, Kawasaki 212-8582, Japan \\ Fax: 81-44-520-1255, e-mail: yohachi yamashita@toshiba.co.jp
}

\begin{abstract}
Temperature dependences of poled dielectric constants $\varepsilon_{33}{ }^{\mathrm{T}} / \varepsilon_{0}$ of $[001] \quad 0.70$ $\mathrm{Pb}\left(\mathrm{Mg}_{1 / 3} \mathrm{Nb}_{2 / 3}\right) \mathrm{O}_{3}-0.30 \mathrm{PbTiO}_{3}$ (PMN-PT) single crystals with different poling fields were measured to investigate the cause of the fluctuation of the $\varepsilon_{33}{ }^{\mathrm{T}} / \varepsilon_{0}$. Anomalous $\varepsilon_{33}{ }^{\mathrm{T}} / \varepsilon_{0}>50,000$ at phase-transition temperature between rhombohedral and tetragonal phases (Trt) with a specific poling field (twice coercive field; $E_{c}$ ) were observed in some samples. Those samples also showed higher $\varepsilon_{33}{ }^{\mathrm{T}} / \varepsilon_{0}=7,700$ and piezoelectric constant $d_{33}=2,000 \mathrm{pC} / \mathrm{N}$ at room temperature than those of other samples. Because each sample had the same Curie temperature Tc, the fluctuations are not derived from the variation in crystal composition variation. Easily or not easily polarized crystal parts may exist in the same wafer. And different domain configurations, defects and impurity levels within the wafer may cause the different mobility of polarization. The temperature dependence of $\varepsilon_{33}{ }^{\mathrm{T}} / \varepsilon_{0}$ measurement is an effective method to investigate the cause of the fluctuation of dielectric and piezoelectric properties of single crystal transducer. Thus, the results of this study provide important guidelines for controlling the fluctuation of these properties of single crystal medical array probes.

Key words: dielectric constant, single crystal, lead magnesium niobate titanate, temperature dependence
\end{abstract}

\section{INTRODUCTION}

A one-dimensional (1-D) medical array, when coupled with a beamformer, allows broadband ultrasonic pulses to be generated and swept through a body for medical diagnostic imaging. The design of a 1-D array, including the number of elements, pitch, and frequency, is realized taking into consideration the particular clinical application. Relaxor single crystals, such as lead zinc niobate titanate $\mathrm{Pb}\left(\mathrm{Zn}_{1 / 3} \mathrm{Nb}_{2 / 3}\right) \mathrm{O}_{3}-\mathrm{PbTiO}_{3}$ (PZN-PT) and lead magnesium niobate titanate $\mathrm{Pb}\left(\mathrm{Mg}_{1 / 3} \mathrm{Nb}_{2 / 3}\right) \mathrm{O}_{3}-\mathrm{PbTiO}_{3}$ (PMN-PT) near the morphotropic phase boundary (MPB) composition with domain engineering configuration are very attractive for medical transducer owing to their large piezoelectric constant $\left(d_{33}\right)>1,500 \mathrm{pC} / \mathrm{N}$ [1]-[3]. Development of piezoelectric single crystals medical ultrasound probes is at the early stage of mass production [4]-[5]. In the medical array application, one piezoelectric material plate is usually cut into multiple array transducers. Therefore, differences in poled dielectric constant $\left(\varepsilon_{33} \mathrm{~T} / \varepsilon_{0}\right)$ and $d_{33}$ between array transducer channels adversely affect echo image. However, fairly large fluctuations of dielectric and piezoelectric properties exist in the PMN-PT single crystal plate.

To obtain better properties of the piezoelectric single crystals, proper polarization conditions are required. The effects of poling conditions (different poling fields and temperatures) on dielectric and piezoelectric properties of [001]-oriented PMN-PT crystal have been investigated [6]-[11]. Guo et al. reported dielectric constant of PMN-PT as a function of temperature. With the poling field increases, the dielectric constant peak temperature at phase transition between ferroelectric rhombohedral and tetragonal (Trt) shifts toward lower temperatures and the dielectric constant decreases. Proper poling condition of PMN-PT has two small dielectric peaks at the Trt. It indicates the rhombohedral-monoclinic and monoclinic-tetragonal phase transitions occur [7]-[8].

Other research of poling properties of [001]-oriented PMN-PT in the MPB was reported. The Trt and Curie temperature $(T \mathrm{c})$ were changed by composition ratio. Some specific composition samples show anomalous dielectric constant peak at Trt. As the PT content increased toward the MPB, Trt shifts lower and Tc shifts to higher temperature. In addition, in samples closer to the MPB, dielectric anomalies occur at $T \mathrm{rt}$ that are larger than the value at $T \mathrm{c}$ [11].

However, fluctuation of the dielectric and piezoelectric properties of the PMN-PT large wafer grown in [001] direction and its mechanism have not been studied so far.

The purpose of this study is to investigate the $\varepsilon_{33}{ }^{\mathrm{T}} / \varepsilon_{0}$ and $d_{33}$ fluctuation of [001]-oriented PMN-PT single crystal wafer for the medical probe application.

\section{EXPERIMENTS}

The $0.70 \mathrm{~Pb}\left(\mathrm{Mg}_{1 / 3} \mathrm{Nb}_{2 / 3}\right) \mathrm{O}_{3}-0.30 \mathrm{PbTiO}_{3}$ (70 PMN-30 $\mathrm{PT}$ ) single crystal wafer used in this work was grown in [001] direction by a modified Bridgman method. The crystal oriented along the [001] direction was prepared with $50 \mathrm{~mm}$ diameter and $0.28 \mathrm{~mm}$ thickness wafer and 


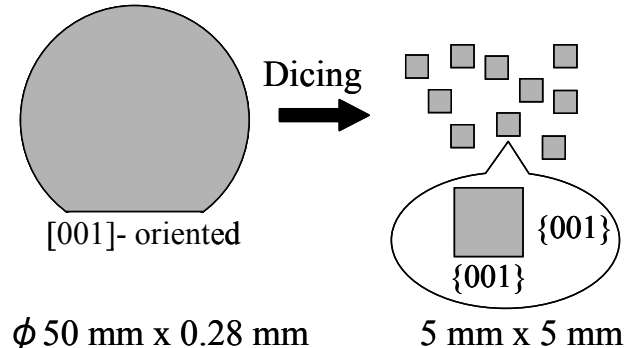

Fig. 1 PMN-PT samples.

coated with sputtered chrome and gold electrodes. Rectangular plate samples of $5 \times 5 \times 0.28 \mathrm{~mm}$ were cut from the wafer and measured as shown in Fig. 1.

First, a coercive field $\left(E_{c}\right)$ of the PMN-PT sample was measured by the Sawyer-Tower circuit at room temperature RT $\left(25^{\circ} \mathrm{C}\right)$. Then samples were heated up to $200{ }^{\circ} \mathrm{C}$ to depolarize them and poling was carried out at RT for 5 minutes with 3 kinds of electric field determined from the value of $E_{c}: 2.5$ (equal to $E_{c}$ ), 5.0 (twice of $E_{c}$ ) and $7.5\left(3\right.$ times of $\left.E_{c}\right) \mathrm{kV} / \mathrm{cm}$.

The piezoelectric constants $d_{33}$ were measured by a $d_{33}$ meter (Institute of Acoustics Academia Sinica, Model ZJ-3D). Dielectric constants as a function of temperature were measured using an HP4192A impedance analyzer at $1 \mathrm{~V}$ at $1 \mathrm{kHz}$. Samples were heated from RT to $190{ }^{\circ} \mathrm{C}$ at the rate of $1{ }^{\circ} \mathrm{C} / \mathrm{min}$.

Each time before poling field was applied, the samples were thermally depolarized by heating them to $200{ }^{\circ} \mathrm{C}$.

\section{RESULTS AND DISCUSSION}

Figure 2 shows a ferroelectric hysteresis loop displayed at $1 \mathrm{~Hz}$ of triangle wave at RT. The $E_{c}$ is determined from this loop. There are some $E_{c}$ fluctuations in the samples from 2.2 to $2.5 \mathrm{kV} / \mathrm{cm}$, and remanent polarizations $P_{r}$ were 21 to $23 \mu \mathrm{C} / \mathrm{cm}^{2}$. The poling fields were determined in multiple numbers of the $E_{c}$ of $2.5 \mathrm{kV} / \mathrm{cm}$.

Figure 3 shows $d_{33}$ of each poling field at RT. At the poling field of $5.0 \mathrm{kV} / \mathrm{cm}, d_{33} \mathrm{~s}$ of samples are split; high and low. Poled dielectric constants $\varepsilon_{33}{ }^{\mathrm{T}} / \varepsilon_{0}$ at RT show the same tendency. So we call the samples H- $\varepsilon$ and L-e. The $d_{33}$ of $\mathrm{H}-\varepsilon$ sample slightly decrease at $7.5 \mathrm{kV} / \mathrm{cm}$ poling field.

Figure 4 shows temperature dependences of poled dielectric constant (TDD) of the 3 kinds of poling field of crystal samples. At the $2.5 \mathrm{kV} / \mathrm{cm}$ poling field shown in Fig. 4 (a), the shape of the curves and dielectric constant are similar. And at RT, $\varepsilon_{33} \mathrm{~T} / \varepsilon_{0}$ was 5,700 . The temperature peak of phase transition $(\mathrm{Trt})$ was $100{ }^{\circ} \mathrm{C}$.

Fig. 4 (b) shows $5.0 \mathrm{kV} / \mathrm{cm}$ poling field of the same samples. As in the case of the results of $d_{33}$, there was a split into two groups. Each curve has 2 peaks of Trt; the peaks indicate the phase transitions of rhombohedral to monoclinic temperature ( $T \mathrm{rt} 1$ or $T \mathrm{rm}$ ), and monoclinic to tetragonal temperature (Trt2 or Tmt). The $\mathrm{H}-\varepsilon$ sample shows anomalous large peak of $\varepsilon_{33} \mathrm{~T} / \varepsilon_{0}$ of 53,000 at $90{ }^{\circ} \mathrm{C}$ of Trt1 and smaller peak of 29,800 at $99{ }^{\circ} \mathrm{C}$ of $\mathrm{Trt} 2$. The L- $\varepsilon$ sample shows two peaks of 24,600 and 26,000 at $96{ }^{\circ} \mathrm{C}$ and $102{ }^{\circ} \mathrm{C}$, respectively, but $\operatorname{Trt} 1$ was $6{ }^{\circ} \mathrm{C}$ higher and dielectric peak was not particularly large compared to H- $\varepsilon$ 's. For this $5.0 \mathrm{kV} / \mathrm{cm}$ poling field, $\varepsilon_{33}{ }^{\mathrm{T}} / \varepsilon_{0}$ at RT

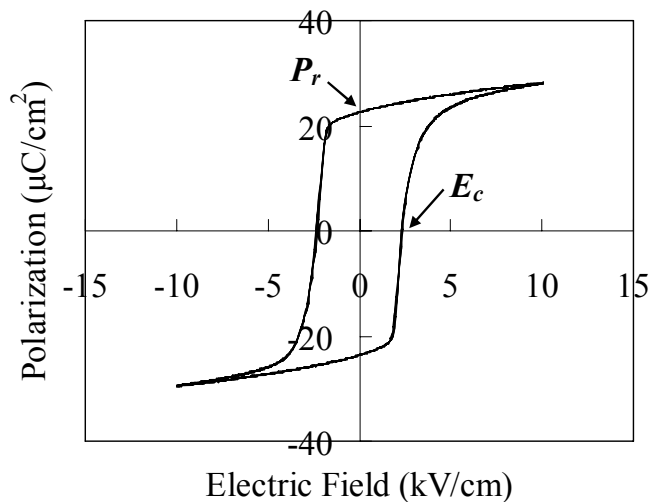

Fig. 2 D-E hysteresis loop at $1 \mathrm{~Hz}$ at room temperature.

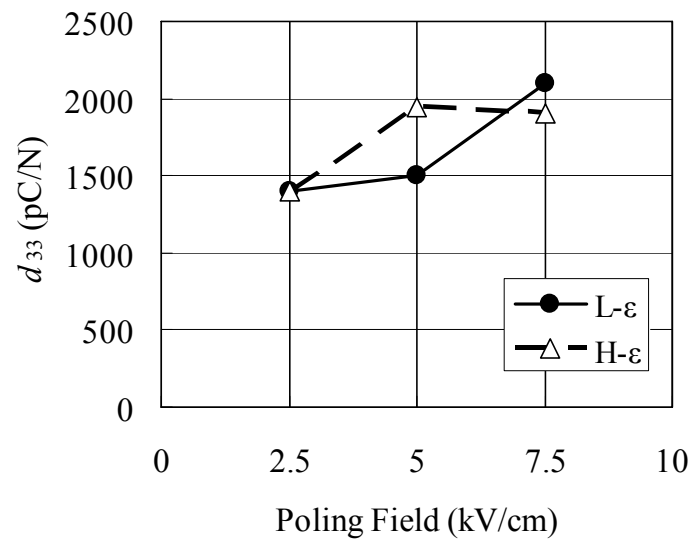

Fig. 3 Poling field dependence of piezoelectric constant $d_{33}$ at room temperature.

was 7,700 of $\mathrm{H}-\varepsilon$ and 6,100 of L- $\varepsilon$. By comparison, the $E_{c}=2.2 \mathrm{kV} / \mathrm{cm}$ of the H- $\varepsilon$ samples were rather smaller than the L-E's of $E_{c}=2.5 \mathrm{kV} / \mathrm{cm}$.

However all samples show the same Curie temperature $(T \mathrm{c})$ of $137{ }^{\circ} \mathrm{C}$. Because $T \mathrm{cs}$ were invariable, the fluctuation of the $\varepsilon_{33}{ }^{\mathrm{T}} / \varepsilon_{0}$ for the samples is not caused by the crystal composition variation. Therefore anomalous dielectric constant at $T$ rt with a specific poling field (twice of $E_{c}$ ) is related to the large dielectric constant fluctuation at RT.

Fig. 4 (c) shows the $7.5 \mathrm{kV} / \mathrm{cm}$ poling field of the same samples. Almost the same curves of TDD are exhibited. Two peaks of Trt are similar, and dielectric constants at RT show small difference; 7,600 of H- $\varepsilon$ and 7,700 of L-E.

The dielectric loss measurement at $1 \mathrm{kHz}$ from $20{ }^{\circ} \mathrm{C}$ to $200{ }^{\circ} \mathrm{C}$ revealed that those values were less than $10 \%$ in the $T \mathrm{rt}$ and $T \mathrm{c}$ regions. So the $\varepsilon_{33}{ }_{\mathrm{T}} / \varepsilon_{0}$ values reported here are reliable.

Figure 5 shows $\varepsilon_{33}{ }^{\mathrm{T}} / \varepsilon_{0}$ at RT and Trt1 of each poling field from the measurements of TDDs. The tendency of poling field of $\varepsilon_{33} \mathrm{~T} / \varepsilon_{0}$ at both temperatures and $d_{33}$ shown in Fig. 3 are similar. The $\varepsilon_{33}{ }^{\mathrm{T}} / \varepsilon_{0} \mathrm{~S}$ and $d_{33}$ of $\mathrm{H}-\varepsilon$ samples decrease in the case of $7.5 \mathrm{kV} / \mathrm{cm}$ poling field. Over-poling of the crystal may occur [10].

From Figs. 3 and 5, it is clear that the $d_{33}$ and $\varepsilon_{33}{ }^{\mathrm{T}} / \varepsilon_{0}$ of the PMN-PT are proportionally correlated as shown 

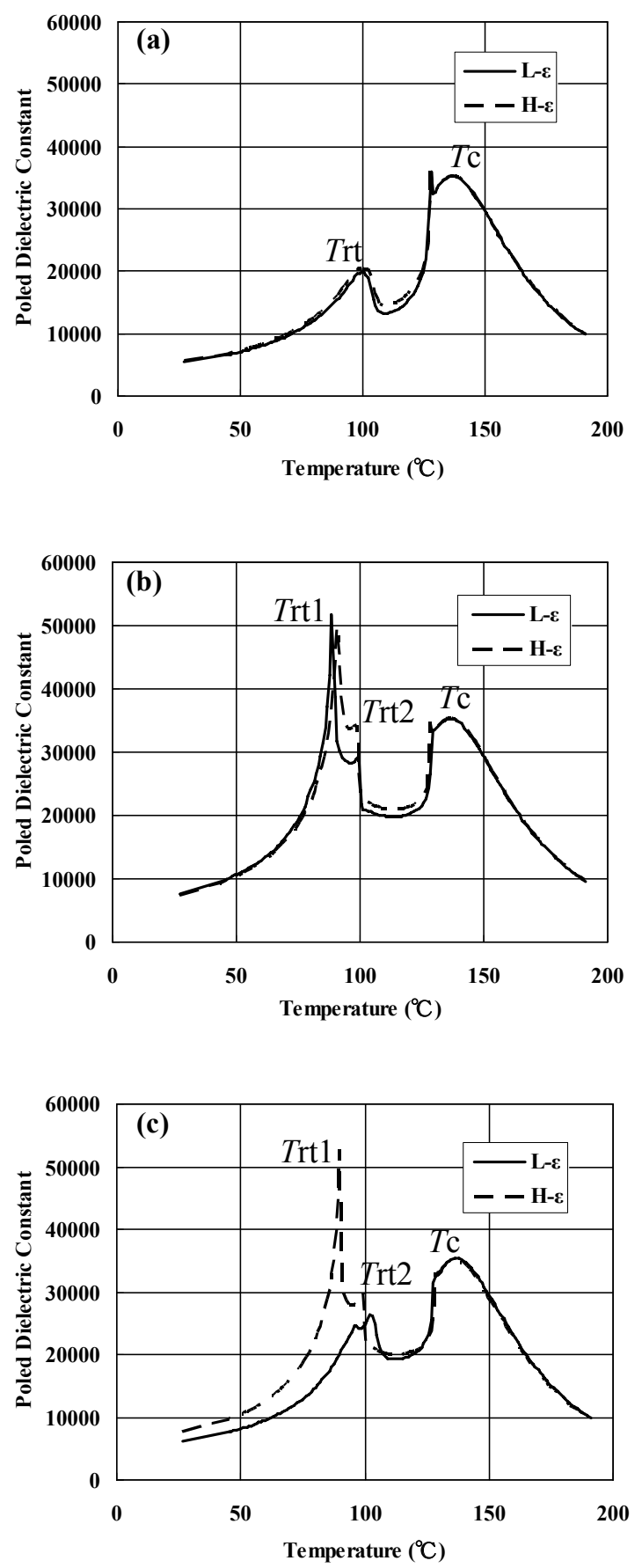

Fig. 4 Temperature dependences of poled dielectric constant with different poling field, (a) $2.5 \mathrm{kV} / \mathrm{cm}$, (b) $5.0 \mathrm{kV} / \mathrm{cm}$, and (c) $7.5 \mathrm{kV} / \mathrm{cm}$.

by previous research [13]. Those differentiations occurred in the samples from same PMN-PT crystal wafer.

Figure 6 presents schematic images of the mechanism of the fluctuation. Some of the types of domain configurations of piezoelectric crystals are shown in Fig. 6 (a). Distributed dispersion of domains exists in the PMN-PT near the MPB. The mobility of the polarization inversion and domain walls by poling field vary

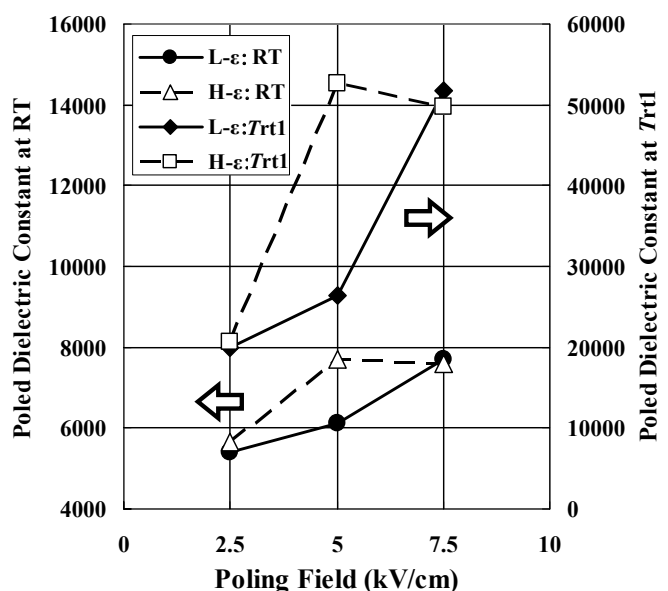

Fig. 5 Poling field dependence of dielectric constant at RT and phase transition temperature Trt1.

(a)
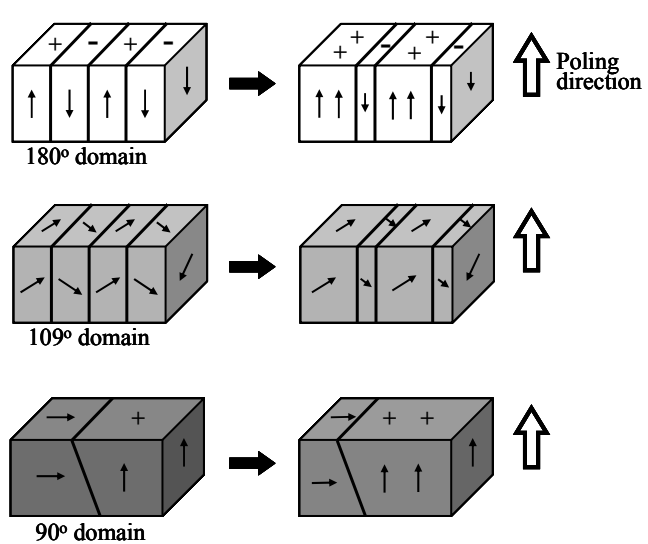

(b)

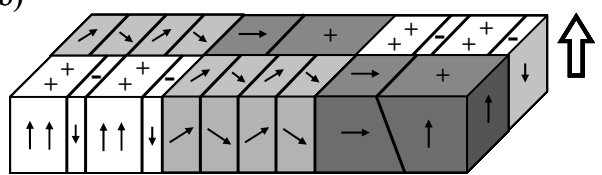

Fig. 6 Schematic images of the mechanism of fluctuations of dielectric and piezoelectric properties, (a) Domain wall moving and (b) Poling reversal of the intermediate state.

depending on domain type. Since domain reversal of $180^{\circ}$ of the MPB composition of relaxor single crystal occurs without volume change, it occurs an early stage of polarization compared to other domains; $71^{\circ}, 90^{\circ}$, and $109^{\circ}$. In the later stage of polarization with higher poling voltage, these domain reversals start a step by step and may create new domain walls. Wada reported that domain walls vicinity of $\mathrm{BaTiO}_{3}$ crystal poses high $d_{33}$ compared to other portion [14]. These domain wall region quantity increasing may contribute the high piezoelectric and dielectric properties of the PMN-PT single crystal. Partial defect and impurities may also influence the polarization. Therefore, easily or not easily polarized crystal parts may exist in the same wafer as previously reported [15]. The fact that $\mathrm{H}-\varepsilon$ samples 
showed lower $E_{c}$ of $2.2 \mathrm{kV} / \mathrm{cm}$, indicates this mechanism.

High $\varepsilon_{33}{ }^{\mathrm{T}} / \varepsilon_{0}$ at room temperature of piezoelectric material is also derived from the shift of $T \mathrm{rt}$ and/or $T \mathrm{c}$ to lower temperature. However, from the result of the temperature dependence of $\varepsilon_{33}{ }^{\mathrm{T}} / \varepsilon_{0}$, an anomalous large peak of $\varepsilon_{33}{ }^{\mathrm{T}} / \varepsilon_{0}$ is the main cause of the fluctuation of dielectric and piezoelectric properties of the PMN-PT.

\section{CONCLUSIONS}

Fluctuations of poled dielectric constant and piezoelectric constant $d_{33}$ at RT of $0.70 \mathrm{PMN}-0.30 \mathrm{PT}$ single crystal wafer were investigated and the following conclusions are reached:

(1) Anomalous poled dielectric constant $\varepsilon_{33}{ }^{\mathrm{T}} / \varepsilon_{0}$ (> $50,000)$ at phase-change temperature $T \mathrm{rt}$ with a specific poling field (twice coercive field) is related to the large $\varepsilon_{33}{ }^{\mathrm{T}} / \varepsilon_{0}$ and $d_{33}$ fluctuation at room temperature.

(2) Because each sample has the same Curie temperature, these fluctuations are not derived from the crystal composition variation.

(3) Easily or not easily polarized crystal parts may exist in the same wafer.

(4) Different domain configurations, defects, and impurity levels within the wafer may cause the different mobility of polarization.

Therefore, the temperature dependence of $\varepsilon_{33}{ }^{\mathrm{T}} / \varepsilon_{0}$ measurement for PMN-PT single crystal is an effective method to investigate the cause of the fluctuation of dielectric and piezoelectric properties of single crystal transducer for medical array probe.

\section{REFERENCES}

[1] J. Kuwata, K. Uchino, and S. Nomura, Jpn. J. Appl. Phys., 21, 1298-302 (1982).

[2] T. R. Shrout, Z. P. Chang, N. Kim, and S. Markgraf, Ferroelectrics Letter, 12, 63-69 (1990).

[3] S. Wada and T. Tsurumi, Key Engineering Materials, 214-215, 9-14 (2002).

[4] S. Saitoh, M. Izumi, S. Shimanuki, S. Hashimoto, and Y. Yamashita, Ultrasonic Probe, U.S. Patent 5,295,487 (Feb. 09, 1994).

[5] S. M. Rhim, H. Jang, S. Kim, and S. G. Lee, Proc. IEEE Ultrason. Sympo., 1115-19 (2002).

[6] E. V. Colla, N. K. Yushin, and D. Viehland, J. Appl. Phys., 83, 3298-305 (1998).

[7] X. Zhao, B. Fang, H. Cao, Y. Guo, and H. Luo, Mater. Sci \& Engineer., B96, 253-62 (2002).

[8] Y. Guo, H. Luo, D. Ling, H. Xu, T. He, and Z. Yin, J. Phys. Condens. Matter, 15, L77-82 (2003) .

[9] D. Viehland and G-F. Li, J. Appl. Phys., 96, 3379-81 (2004).

[10] Z. Feng, X. Zhao, and H. Luo, Mater. Res. Bullet., 41, 1133-37 (2006).

[11] A. Sehirlioglu, D. A. Payne, and P. Han, J. Appl. Phys., 99, 064101 (2006).

[12] K. K. Rajan, M. Shanthi, W. S. Chang, J. Jin, and L. C. Lim, Sensor \& Actuators, A 133, 110-16(2007).

[13] J. Tian, P. Han, D. A. Payne, Trans. Ultrason. Ferroelect. Freq. Contr., 54, No. 9, 1895-902 (2007).

[14] S. Wada, Ceramics Japan, 43, No. 8, 639-647 (2008).

[15] A. A. Bokov and Z-G. Ye, Apply. Phys. Lett., 92, 082901 (2008).

(Received December 25, 2009; Accepted January 31, 2010) 